Communications in Physics, Vol. 28, No. 2 (2018), pp. 163-168

DOI:10.15625/0868-3166/28/2/11673

\title{
CONSTRUCTING ELECTRONIC PHASE DIAGRAM FOR THE HALF-FILLED HUBBARD MODEL WITH DISORDER
}

\author{
HOANG ANH TUAN ${ }^{\dagger}$ AND NGUYEN THI HAI YEN \\ Institute of Physics, Vietnam Academy of Science and Technology \\ ${ }^{\dagger} E$-mail: hatuan@iop.vast.ac.vn
}

Received 7 March 2018

Accepted for publication 15 April 2018

Published 16 June 2018

\begin{abstract}
The electronic phase diagram of strongly correlated systems with disorder is constructed using the typical-medium theory. For half-filled system, the combination of the linearized dynamical mean field theory and equation of motion approach allows to derive the explicit equations determining the boundary between the correlated metal, Mott insulator, and Anderson insulator phases. Our phase diagram is consistent with those obtained by the more sophisticated methods.
\end{abstract}

Keywords: phase diagram, disordered Hubbard model, Anderson insulator .

Classification numbers: 71.27.+a .

\section{INTRODUCTION}

The development of the quantum theory of solids led to the detection of different mechanisms being able to cause a metal - insulator transition (MIT). The correlation induced metal insulator transitions are called Mott transition. An important model to study electron correlations and Mott transition is the famous Hubbard model (HM). On the other hand, disorder in solids, such as impurities or vacancies, was found to strongly modify band theory predictions. In 1958, Anderson showed in his analysis of a disordered tight binding model that a sufficient amount of disorder hinders the diffusion of particles [1]. Coherent backscattering processes cause a localization of the particle. In particular, the localization of states at the Fermi level induces a metal insulator transition, the Anderson transition.

The interplay of disorder and interactions leads to many important and unexpected effects. Among them, the MIT at non-integer filling, have been found by Byczuk and coworkers by the (C) 2018 Vietnam Academy of Science and Technology 
dynamical mean field theory (DMFT) $[2,3]$. They have shown that at a particular density, equal to the disorder concentration $x$ or $(1+x)$, the interplay between disorder induced band splitting and correlation induced Mott transition gives rise to a new type of MIT in the HM with the binary disorder. A similar result has been found by Monte Carlo simulation [4]. The metal insulator phase diagram as a function of $\Delta$ and $U$, where $\Delta$ is the energy difference between two types of sites at $T=0 K$ have been investigated by mean of the combination of DMFT and coherent potential approximation (CPA) [5,6]. For half filled system, when $\Delta$ is sufficient large, two transitions from a band insulator via a metallic state to a Mott insulator are found with increasing $U$. It is interesting to note that the presence of weak interactions might induce a significant enhancement of transport, while strong interactions contrarily tend to suppress it strongly. However, the above obtained phase diagram remains uncompleted, because the arithmetic average of random oneparticle quantities (e.g. density of states, DOS) calculated within such meanfield theories can not distinguish between extended and localized states and are not critical at the Anderson transition. Finding a proper single particle order parameter for the Anderson localization transition capable of distinguishing between the localized and extended states is a major challenge in the study of disordered electronic systems. In contrast to the arithmetic average, the geometrical average gives a better approximation to the most probable value of the local DOS. Dobrosavljevic and coworkers developed the typical medium theory (TMT) to study disordered systems, where the typical density of states (TDOS), approximated using the geometrical averaging over disorder configurations, is used instead of the arithmetically averaged local DOS [7]. They demonstrated that the TDOS vanishes continuously as the strength of the disorder increases toward the critical point and it can be used as an order for the Anderson localization transition. This scheme uses only one-particle quantities and can be incorporated into the DMFT for disordered electrons in presence of Coulomb correlations [8-10]. Recently, the metal-insulator phase diagram in the half-filled HM with a box disorder has been investigated within the TMT - DMFT with different standard numerical impurity solvers, such as the numerical renormalization group (NRG) method [11], the four boson technique (SB4) [12]. These numerical methods are seen to work well for the model, but each method has its limitations and all of them are computationally expensive, with their applications being strongly limited by available computer resources.

In this paper, the TMT-linearized DMFT and the equation of motion approach are used to obtained the nonmagnetic ground state phase diagram of the half-filled HM with disorder. The equations determining the boundary between the correlated metal, Mott insulator, and Anderson insulator phases are derived analytically. Our results are consistent with those obtained from the TMT-DMFT with the NRG and the SB4.

\section{MODEL AND SOLVING METHOD}

We consider a half-filled Hubbard model with random site energies, as given by the Hamiltonian

$$
H=-t \sum_{<i j>\sigma}\left(a_{i \sigma}^{\dagger} a_{j \sigma}+\text { H.c. }\right)+\sum_{i \sigma} \varepsilon_{i} n_{i \sigma}+\sum_{i} U_{i} n_{i \uparrow} n_{i \downarrow},
$$

where $a_{i \sigma}\left(a_{i \sigma}^{\dagger}\right)$ annihilates (creates) an electron with spin $\sigma$ at site $i, n_{i \sigma}=a_{i \sigma}^{\dagger} a_{i \sigma}$ and the sum $\langle i j\rangle$ is the sum over nearest neighbor sites of a Bethe lattice. $t$ is the hopping amplitude, and $U$ is the on-site Coulomb repulsion. The random on-site energies $\varepsilon_{i}$ follow a distribution $P\left(\varepsilon_{i}\right)$, 
which is assumed to be uniform and have width $\Delta$, i.e. $P\left(\varepsilon_{i}\right)=\Theta\left(\Delta / 2-\left|\varepsilon_{i}\right|\right) / \Delta$, with $\Theta$ as the step function. The parameter $\Delta$ is a measure of the disorder strength.

TMT-DMFT maps the lattice problem onto an ensemble of effective single-impurity Anderson Hamiltonian with different $\varepsilon_{i}$ :

$$
H_{i m p}=\sum_{\sigma}\left(\varepsilon_{i}-\mu\right) n_{i \sigma}+U n_{i \uparrow} n_{i \downarrow}+\sum_{k \sigma} \varepsilon_{k \sigma} c_{k \sigma}^{+} c_{k \sigma}+\sum_{k \sigma}\left(V_{k} c_{k \sigma}^{+} a_{i \sigma}+V_{k}^{*} a_{i \sigma}^{+} c_{k \sigma}\right) .
$$

Here $\mu$ is the chemical potential, $V_{k}$ and $\varepsilon_{k}$ are the hybridization matrix element and the dispersion of the auxiliary bath fermions $c_{k \sigma}$, respectively. The effective parameters $\varepsilon_{k}$ and $V_{k}$ enter the hybridization function as

$$
\eta(\omega)=\sum_{k} \frac{V_{k}^{2}}{\omega-\varepsilon_{k}} .
$$

Given the hybridization function $\eta(\omega)$, one first needs to solve the local impurity models and compute the local density of states (LDOS) $\rho\left(\omega, \varepsilon_{i}\right)=-\mathfrak{I} G\left(\omega, \varepsilon_{i}\right) / \pi$. From the $\varepsilon_{i}$-dependent LDOS one can obtain either the geometrically averaged LDOS $\left.\rho_{\text {geom }}(\omega)=\exp \left[<\ln \rho\left(\omega, \varepsilon_{i}\right)\right\rangle\right]$ or the arithmetically averaged LDOS $\rho_{\text {arith }}(\omega)=<\rho\left(\omega, \varepsilon_{i}\right)>$, where $<O\left(\varepsilon_{i}\right)>=\int d \varepsilon_{i} P\left(\varepsilon_{i}\right) O\left(\varepsilon_{i}\right)$ is an arithmetic mean of $O\left(\varepsilon_{i}\right)$. The lattice Green function is given by the Hilbert transform

$$
G(\omega)=\int d \omega^{\prime} \frac{\rho_{\alpha}\left(\omega^{\prime}\right)}{\omega-\omega^{\prime}}
$$

where the subscript $\alpha$ stands for either "geom" or "arith."

The Anderson-Hubbard model (AHM) (1) is solved for a bare semielliptic DOS for the Bethe lattice, $\rho_{0}(\varepsilon)=4 \sqrt{1-4(\varepsilon / W)^{2}} /(\pi W)$. For this DOS a simple algebraic relation between the local Green function $G(\omega)$ and the hybridization function $\eta(\omega)=W^{2} G(\omega) / 16$. In the halffilled band case, the ground state properties are determined by the character of quantum state at the Fermi level $(\omega=0)$. The metallic phase is characterized by $\rho_{\text {geom }}(0)>0$, the Mott insulator (hard gap) occurs when $\rho_{\text {arith }}(0)=0$, and the system is in the Anderson insulator (gapless) when $\rho_{\text {geom }}(0)=0, \rho_{\text {arith }}(0)>0$.

In this paper the impurity model (2) is solved at zero temperature by the decoupling the equations of motion at the second order. Our study is restricted to the paramagnetic case at half-filling: $\mu=U / 2$ and $\left\langle n_{\uparrow}\right\rangle=\left\langle n_{\downarrow}>\right.$. To qualitatively obtain the metal-insulator phase diagram of the system, we make the following approximation for the impurity Green function:

$$
\begin{aligned}
G\left(\omega, \varepsilon_{i}\right) & =\frac{1 / 2}{\omega-\varepsilon_{i}+U / 2-\eta(\omega)+U \eta(\omega)\left[\omega-\varepsilon_{i}-U / 2-3 \eta(\omega)\right]^{-1}} \\
& +\frac{1 / 2}{\omega-\varepsilon_{i}-U / 2-\eta(\omega)-U \eta(\omega)\left[\omega-\varepsilon_{i}+U / 2-3 \eta(\omega)\right]^{-1}} .
\end{aligned}
$$

We note that Eq. (5) is exact in the case $U=0$ [7] and for $U \neq 0, \varepsilon_{i}=0$ is known as the (full) Hubbard III approximation of the half-filled Hubbard model [13].

To proceed further we use the linearized DMFT (L-DMFT). In the band center, the Green function is purely imaginary, $G(0)=-i \pi \rho_{\alpha}(0)$ due to a symmetry of $\rho_{\alpha}(\omega)$. Hence the DMFT self-consistency leads to the recursive relation $G(0)^{(n+1)}=-i \pi W^{2} \rho_{\alpha}^{(n)}(0)$, where the left hand side in the $(n+1)$ th iteration step is given by the result from the $(n)$ th iteration step. Using Eq. 
(5) and expanding them with respect to small $\rho_{\alpha}^{(n)}(0)$ we can obtain the DMFT recursive relation

$$
\rho_{\alpha}^{(n+1)}\left(0, \varepsilon_{i}\right)=\frac{W^{2}}{16} \rho_{\alpha}^{(n)}(0) Z\left(\varepsilon_{i}\right)
$$

where

$$
Z(\varepsilon)=\frac{\varepsilon^{2}+3 U^{2} / 4}{\left[\varepsilon^{2}-U^{2} / 4\right]^{2}}
$$

The boundary between metallic and insulating phases can be found when condition $\rho_{\alpha}^{(n+1)}(0)=$ $\rho_{\alpha}^{(n)}(0)$ is satisfied. Then, by using Eqs. (6) and (7) and evaluating the averaged on $\varepsilon_{i}$, we finally obtain the equations determining the curves $\Delta=\Delta(U)$; i.e.,

$$
1=\frac{W^{2}}{16} \exp \left[I_{\text {geom }}(U, \Delta)\right]
$$

where

$$
\begin{array}{r}
I_{\text {geom }}(U, \Delta)=2+\ln \left[3(U / 2)^{2}+(\Delta / 2)^{2}\right]+\frac{2 \sqrt{3} U}{\Delta} \arctan \left(\frac{\Delta}{\sqrt{3} U}\right) \\
-2 \ln \left|(U / 2)^{2}-(\Delta / 2)^{2}\right|-\frac{2 U}{\Delta} \ln \frac{\Delta+U}{|\Delta-U|},
\end{array}
$$

for L-DMFT with geometrical averaging, and

$$
1=\frac{W^{2}}{16} I_{\text {arith }}(U, \Delta)
$$

where

$$
\left.I_{\text {arith }}(U, \Delta)\right]=\frac{2}{(U / 2)^{2}-(\Delta / 2)^{2}}+\frac{2}{\Delta U} \ln \frac{\Delta+U}{|\Delta-U|},
$$

for L-DMFT with arithmetical averaging.

Equations (8) - (11), which determine the electronic phase diagram $U-\Delta$ of the half-filled Anderson - Hubbard model, are our main result.

\section{RESULTS AND DISCUSSION}

Through this work we set $W$ as the unit of the energy. Solutions of Eqs. (8) - (11) are shown in Fig. 1. Our phase diagram in the interaction - disorder $(U-\Delta)$ plane is generally consistent with that of Refs. [11,12]. Three different phases are found to take place: the metallic phase is identified for small values of $U$ and $\Delta$, the Mott insulator phase stabilizes as $U$ increases, and the Anderson localization overcomes for large $\Delta$. In addition, the presence of disorder increases the critical interaction from $U_{c}(\Delta=0)=\sqrt{3} / 2$ for the Mott-Hubbard MIT. On the other hand, the critical disorder strength for Anderson localization increases for weak interaction $(0 \leq U \leq 1.7)$ starting from the exact value $\Delta_{c}(U=0)=e / 2 \approx 1.36$, then is suppressed by strong interactions. We note that at weak disorder $(0 \leq \Delta \leq 0.7)$ L-DMFT with geometrical and arithmetical averaging give the same results, while at strong disorder $(\Delta>2.0)$ the border between two types of insulators approaches to the line $\Delta=U$, which well agrees with those of Ref. [12].

We find also that our phase diagram for the Anderson - Hubbard model is similar to the phase diagram for the Anderson-Falicov- Kimball model (AFKM) solved within TMT-DMFT in 


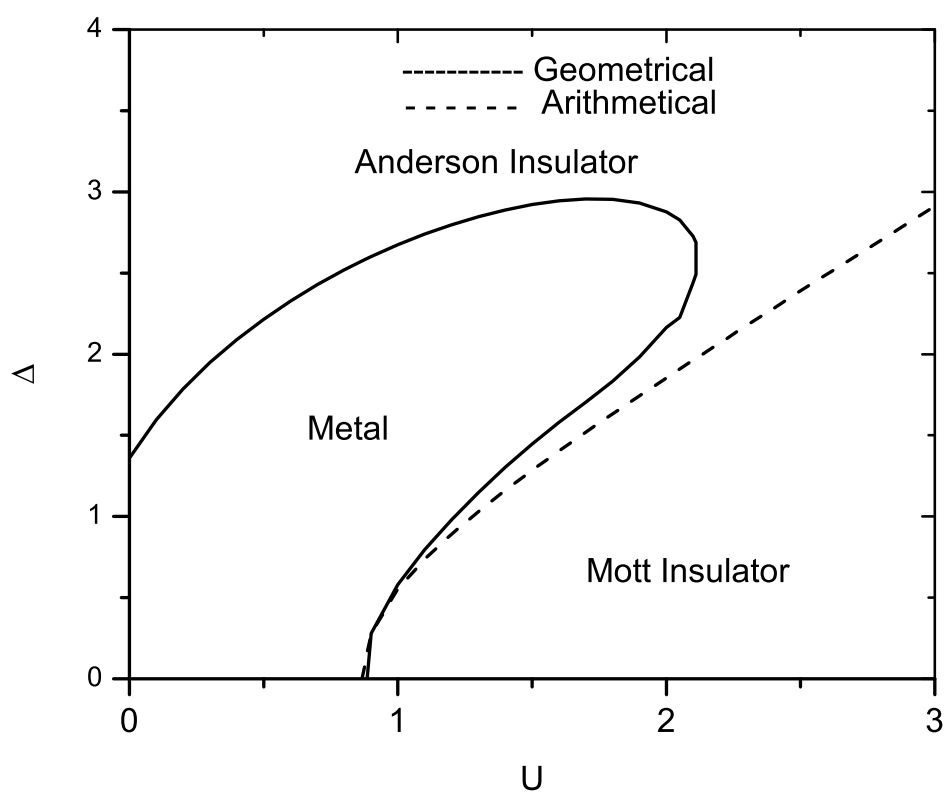

Fig. 1. Electronic ground state phase diagram of the Anderson - Hubbard model at half filling, obtained from the numerical solution of Eqs. (8) - (11). Energy parameters $U, \Delta$ are in energy unit set by $W=1$.

Ref. [8]. However, the metallic region for the AHM is considerably enlarged in comparison with those for the AFKM.

\section{CONCLUSION}

In summary, we have used the equation of motion method as an impurity solver for LDMFT with geometrical and arithmetical averaging to construct the nonmagnetic ground state phase diagram of the AHM at half-filling. This approach allows to derive the explicit equations determining the boundary between the correlated metal, Mott insulator, and Anderson insulator phases. Our results are consistent with those obtained from the TMT-DMFT with the NRG and the SB4. This work demonstrates that the equation of motion approach is a simple, but reliable, impurity solver for constructing the diagram phase in the correlated systems with disorder. In particular, it can be applied to the system with the local ionic energy following the Gaussian and Lorentzian probability distributions or/and with Coulomb disorder. This is left to a future work.

\section{ACKNOWLEDGMENTS}

This research is funded by National Foundation for Science and Technology Development (NAFOSTED) under Grant No. 103.01-2017.56. 


\section{REFERENCES}

[1] P. W. Anderson, Phys. Rev. 109 (1958) 1492.

[2] K. Byczuk, M. Ulmke, and D.Vollhardt, Phys. Rev. Lett. 90 (2003) 196403.

[3] K. Byczuk, M. Ulmke, Eur. Phys. J. B 45 (2005) 449.

[4] N. Paris et al., Phys. Rev. B 75 (2007) 165113.

[5] M. Balzer, M. Potthoff, Physica B 359-361 (2005) 768.

[6] P. Lombardo et al., Phys. Rev. B 74 (2006) 085116.

[7] V. Dobrosavljevic et al., Eur. Phys. Lett. 62 (2003) 76.

[8] K. Byczuk, Phys. Rev. B 71 (2005) 205105.

[9] R. D. B. Carvalho and M. A. Gusmao, Phys. Rev. B 87 (2013) 085122.

[10] R. D. B. Carvalho et al., Eur. Phys. J. B 87 (2014) 160.

[11] K. Byczuk et al., Phys. Rev. Lett. 94 (2005) 056404.

[12] M. C. O. Aguiar et al., Phys. Rev. Lett. 102 (2009) 156402.

[13] J. Hubbard, Proc. Roy. Soc. London A 281 (1964) 401. 